\title{
Traduction du discours politique entre spécialisation et inclusion
}

\section{Translation of Political Discourse between Specialization and Inclusiveness}

\author{
Fatima Zohra Chouarfia \\ Université d'Oran 1 Ahmed Benbella, Algérie \\ chouarfia.fz@hotmail.com
}

\begin{abstract}
Translation of political discourse is one of specialized translation central issues. In this kind of translation, not only are translators required to be familiar with both terminology and phraseology of the field in question, they are also compelled to be a walking encyclopedia due to the diversity of topics that could be dealt with in such texts. Thus, the translation of political discourse proves to be an uphill battle putting a huge responsibility on the translator's shoulders who must be ultracautious when trying to choose the most appropriate and accurate equivalents, for such texts are highly sensitive and any tiny mistake can lead to conflicts, even wars, between nations. The scope of this paper is to examine the process of this type of translation and explore the dilemma of whether or not to consider the translation of political discourse as a kind of specialized translation.
\end{abstract}

Keywords: translation of political discourse - specialized translation - media translation - linguistic and extralinguistic knowledge.

\section{INTRODUCTION}

Le discours politique est le discours le plus répandu chez le commun des mortels. La politique prend le dessus et affecte la vie de tous. Selon Aristote, « l'homme est par nature un animal politique ». Qu'il le devienne ou qu'il le soit de nature, il est politique et social, s'intéresse à ce qui se passe autour de lui et dans le monde et aime le comprendre en sa propre langue d'où la nécessité de la traduction. Touchant les différents aspects de la vie, le discours politique se veut un discours particulier qui vise les masses. Aussi sensible, délicat et inclusif 
qu'il soit, le discours politique impose au traducteur un traitement minutieux de ses composants. Dans cet article, nous allons dans un premier temps revenir sur la notion du discours politique. Ensuite, nous mettrons l'accent sur le processus de la traduction des textes politiques afin de mieux comprendre la nature de ce genre de traduction.

\section{TRADUCTION DU DISCOURS POLITIQUE}

\subsection{DISCOURS POLITIQUE}

Avant d'entamer la traduction du discours politique, il convient d'abord de définir ce que nous entendons par l'appellation «discours politique ». La réponse la plus simple serait de dire qu'il s'agit d'un discours qui traite de la politique. Toutefois, la notion de discours demeure plus ou moins ambiguë jusqu'à nos jours; il est peu probable de tomber sur une définition unanime du terme. La tradition veut que le discours soit réservé à l'oralité et le texte à l'écrit. Or, cette distinction ne tient plus. Selon Chilton \& Schäffner, le discours aurait trois acceptions. Premièrement, le discours pourrait généralement renvoyer à des énoncés en temps réel. Deuxièmement, il peut faire référence à un certain nombre d'énoncés en temps réel perçus comme un seul évènement linguistique (ex. débat politique). Troisièmement, le discours peut être perçu comme la totalité des énoncés dans une société considérée comme étant une entité autonome en évolution (Chilton \& Schäffner, 2002 : 18). Ceci dit, le discours est une pratique sociale (Fairclough, $2001: 18$ ), c'est cet ensemble de déclarations possibles à propos d'une région donnée, qui organise et structure la manière dont on peut parler d'un sujet, un objet ou un processus particuliers. Ainsi, le discours fournit des descriptions, des règles, des permissions et des interdictions des actions sociales et individuelles (Fowler, $1991: 42$ ).

On ne peut faire de la politique sans avoir recours à la langue, les deux sont comme des jumeaux siamois indissociables. Les exemples en sont multiples; déclarer une guerre est un acte linguistique, constituer des groupes sociaux par le biais de la langue fait partie de la politique, pour ne citer que ceux-là. Le discours politique est donc une "forme complexe de l'activité humaine" (Chilton \& Schäffner, 1997 : 207) qui recouvre une panoplie d'actions politiques discursives telles que mener une campagne électorale, l'adoption de lois, la prise de décision, etc. (van Dijk, 1997 : 18). Les traités multilatéraux, les éditoriaux, les conférences de presse et les interviews avec les politiciens, les débats parlementaires, les régulations gouvernementales, etc. sont d'autres types du discours politique. 


\subsection{PROCESSUS DE LA TRADUCTION DU DISCOURS POLITIQUE}

Le grand public prend connaissance des décisions et positions politiques à travers les médias. Si la langue est un outil primordial pour faire de la politique, les médias contribuent, à leur tour, à la diffusion de la cognition politique et des idéologies. Etant des moyens de communication qui visent à démocratiser l'information, tout en respectant les élites politiques et intellectuelles ainsi que les pouvoirs économiques qui les gouvernent, les médias recontextualisent les textes afin de servir une idéologie ou une politique rédactionnelle données. La recontextualisation peut avoir lieu au sein d'une seule langue, mais elle est plus flagrante en traduction vu les différences culturelles et idéologiques qui peuvent exister entre les langues-cultures, sans parler de la politique de l'institution médiatique cible.

Les agences de presse sont généralement la source première de l'information que les médias diffusent. Encore faut-il rappeler que les médias disposent d'un corpus diversifié puisqu'ils touchent à tout. En outre, les informations proviennent des quatre coins du monde d'où la nécessité de la traduction. Toutefois, des agences de presse à l'instar de Reuters et l'AFP ne recrutent pas de traducteurs ; elles embauchent des journalistes qui connaissent au moins deux langues en plus de leur(s) langue(s) maternelle(s) (Bielsa \& Basssnett, 2009 : 81) et ce en raison des tâches que doivent accomplir les personnes occupant des postes à un tel endroit. Les journalistes sont sélectionnés selon leur maitrise des différentes langues étrangères outre leur savoir-faire en matière de rédaction et de production médiatique. Avec le temps, ces journalistes apprennent à devenir traducteurs et vice versa ; certaines agences recrutent des traducteurs qui font du journalisme une fois sur le terrain (Bielsa \& Basssnett, 2009: 85-86). Alors, qu'on soit traducteur-journaliste ou journaliste-traducteur, on fait le même travail malgré la formation très distincte des deux. Ainsi, traduire pour une institution médiatique implique être prêt à modifier la version des faits et donner celle qu'impose la hiérarchie pour des raisons variables (culturelles, politiques, idéologiques, etc.)

Quand un discours politique est délivré, les différents médias à travers le monde se précipitent à le traduire et le diffuser à leurs publics. Il subit alors les modifications nécessaires avant d'être mis à la disposition du grand public. Or, il n'est pas sûr que la version fournie soit fiable ou, du moins, de qualité. Manquant de temps et étant obligé de traduire plusieurs textes par jour, le traducteur, et l'interprète (travaillant pour des chaines télévisées) encore plus, peut tomber dans l'erreur, tout à fait humaine mais impardonnable dans ce genre de situation, et l'histoire nous en raconte des histoires à ce propos.

Partant du postulat que le discours politique est destiné au grand public, les politiciens ont tendance à opter pour un vocabulaire plus ou moins accessible 
à tous. Cet accès implique parfois une vulgarisation de certaines notions de la part du rédacteur du discours puisque tout est politique, et l'objectif n'est pas de se vanter de connaitre beaucoup de choses sur plusieurs domaines mais plutôt de s'attaquer aux questions qui préoccupent les destinataires. Hormis le choix lexical minutieux, le discours politique se caractérise par un recours aux références culturelles et religieuses propres au public visé (Schäffner, 1997 : 119-120). En effet, plusieurs textes s'emboitent dans un seul discours. Ce phénomène, mieux connu sous le nom intertextualité, est très commun en ce genre de textes. D'après Barthes, «tout texte est un intertexte; d'autres textes sont présents en lui, à des niveaux variables, sous des formes plus ou moins reconnaissables: les textes de la culture antérieure et ceux de la culture environnante » (Barthes, 1974). L'intertextualité n'est pas un signe de bonne mémoire ni un choix parmi d'autres, elle est inévitable. C'est un processus mental obligatoire qui complète l'expérience textuelle des récepteurs (Riffaterre, $1984: 142)$.

L'identification et la reconnaissance des références se font en fonction des connaissances qu'a le récepteur. Certaines sont plus faciles à repérer, tandis que d'autres sont difficilement identifiables. Le traducteur/interprète doit être à même de les identifier et les rendre convenablement en langue cible.

Tableau 1

\begin{tabular}{|c|c|c|c|c|}
\hline Texte source & $\begin{array}{l}\text { Traduction de la } \\
\text { Maison Blanche }\end{array}$ & $\begin{array}{l}\text { Traduction de la chaine } \\
\text { publique égyptienne }\end{array}$ & $\begin{array}{c}\text { Traduction de la } \\
\text { chaine Al Jazeera }\end{array}$ & $\begin{array}{l}\text { Traduction de la } \\
\text { chaine Alarabiya }\end{array}$ \\
\hline $\begin{array}{l}\text { "O mankind! } \\
\text { We have cre- } \\
\text { ated you male } \\
\text { and a female; } \\
\text { and we have } \\
\text { made you into } \\
\text { nations and } \\
\text { tribes so that } \\
\text { you may know } \\
\text { one another." }\end{array}$ & 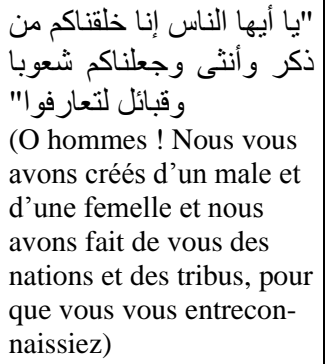 & 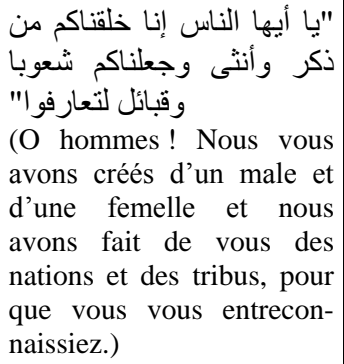 & $\begin{array}{l}\text { "إنا خلقناكم من شعاو قبائل } \\
\text { لتعارفو ا" } \\
\text { (Nous vous avons } \\
\text { créés de nations et } \\
\text { de tribus, pour que } \\
\text { vous vous entre- } \\
\text { connaissiez) }\end{array}$ & $\begin{array}{l}\text { لقد خلقنا الإنسـان لقد خلقتاكم لتعائل لتعارفو أمن } \\
\text { (Nous avons créé l'hom- } \\
\text { me ! Nous vous avons } \\
\text { créé de tribus pour que } \\
\text { vous vous entrecon- } \\
\text { naissiez) }\end{array}$ \\
\hline $\begin{array}{l}\text { "Be conscious } \\
\text { of God and } \\
\text { speak always } \\
\text { the truth." }\end{array}$ & 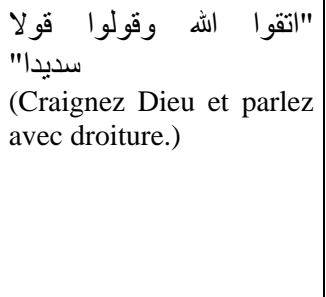 & 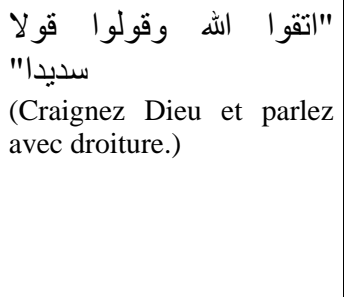 & 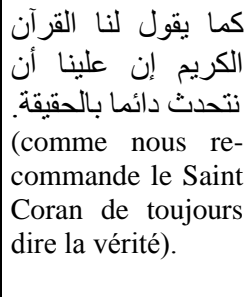 & 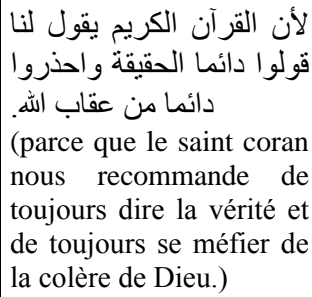 \\
\hline
\end{tabular}


Dans son discours tenu au Caire en 2009, Barack Obama a cité quelques versets coraniques traduits en anglais. Ces derniers apparaissent dans la version arabe de la Maison Blanche et l'interprétation télévisée de la chaine publique égyptienne tels qu'ils figurent dans le Saint Coran (révélé en arabe). Par contre, les interprétations des chaines d'Al Jazeera et Alarabiya ne contiennent que des paraphrases. La tradition arabo-musulmane veut que le Coran soit cité en arabe, soit en sa langue d'origine, exactement tel qu'il a été révélé, une pratique un peu difficile à l'oral vu que la plupart des locuteurs, y compris les interprètes, n'apprennent pas les textes par cœur, ce qui explique le recours à la paraphrase. En postulant que le texte source est unique et que les traductions sont multiples, nous dirons que c'est tout à fait normal que la forme du texte source diffère d'un interprète/traducteur à l'autre sauf que les textes sacrés, surtout s'ils sont cités en leur langue d'origine, doivent apparaître tels qu'ils figurent dans les livres sacrés. Cette contrainte s'avère un calvaire pour les interprètes privés du temps nécessaire de recourir aux textes source, et si dans les cas cités ci-dessus l'interprète travaillant pour la chaine publique égyptienne a réussi à citer le verset correctement c'est que le texte du discours lui a été remis avant le jour J.

\section{TRADUCTION SPÉCIALISÉE OU TRADUCTION GÉNÉRALE?}

Faire de la traduction spécialisée ou de la traduction générale implique avoir affaire à une langue de spécialité ou une langue générale. Or, il est difficile de discerner les frontières entre la langue générale et la langue de spécialité puisque toutes les deux partagent les mêmes règles morphosyntaxiques, seuls le lexique et les dénominations des concepts diffèrent (Lerat, 1988 : 22). Différente de la langue courante, la langue de spécialité se distingue par son vocabulaire technique. Le mot technique englobe tout ce qui se rapporte aux arts, sciences, métiers, certaines activités de loisir, etc. (Dubuc, 2002: 6). De plus, chaque langue de spécialité a ses propres terminologie et phraséologie appartenant à leur tour à un domaine donné. Quand un texte dépasse les frontières de la discipline à laquelle il est censé appartenir, sera-t-on toujours en mesure de parler de spécialisation?

\subsection{DÉFINITIONS}

Avant de déterminer de quel type de traduction il s'agit, il faut passer par l'analyse du texte de départ. Ce que nous entendons par le terme analyse est la lecture et la compréhension du texte source en mettant l'accent sur le(s) registre(s) 
utilisé(s) car ceci déterminera le jargon qu'emploiera le traducteur dans le texte cible. Généralement parlant, une traduction est soit spécialisée soit générale.

Dans la littérature traductologique, les dénominations traduction spécialisée et traduction technique sont souvent employées interchangeablement (Olohan, 2009 : 246) pour désigner toute traduction traitant d'un matériau d'une technicité renvoyant à un domaine spécifique. Or, Daniel Gouadec, à titre d'exemple, fait la distinction entre les deux. Selon lui, la traduction spécialisée est une traduction dont le contenu renvoie à un domaine de travail particulier (Gouadec, 2007: 28), tandis que la traduction technique est une sous-catégorie de la traduction spécialisée qui se rapporte à des domaines tels que l'informatique, les télécommunications, le génie mécanique, etc. (Gouadec, 2007 : 30). Byrne relie, lui aussi, la traduction technique à la technologie et ajoute qu'elle traite plus précisément des textes portant sur des sujets fondés sur les connaissances appliquées des sciences naturelles (Byrne, 2006 : 3). Jean Delisle, quant à lui, propose de distinguer la traduction littéraire de celle des textes pragmatiques et associe ces derniers aux textes véhiculant une information et «dont l'aspect esthétique n'est pas l'aspect dominant » (Delisle, 1980 : 22). Tandis que Daniel Gile fait la distinction entre traduction littéraire, reposant essentiellement sur l'élément esthétique, et celle des textes informatifs où l'information prend le dessus (Gile, 2005 : 4). Aux fins du présent travail, nous définissons la traduction spécialisée comme étant une traduction dont la langue du texte a tendance à être spécifique à un groupe d'experts en un domaine donné nécessitant ainsi des connaissances linguistiques et extralinguistiques spécialisées pour pouvoir être effectuée.

Le concept de spécialisation est subjectif. Chacun a sa propre conception des choses ; ce que l'un considère comme très spécialisé pourraît paraître acceptable ou banal pour un autre. De plus, les disciplines sont liées l'une à l'autre et la catégorisation qui se fait selon le contenu du texte n'est pas très efficace puisque certains textes, notamment les textes politiques qui se trouvent à la croisée des chemins de plusieurs disciplines en l'occurrence les sciences politiques, la sociologie, la psychologie, la linguistique et la théologie (Dorna, 1995 : 132), traitent rarement un seul sujet dans un même texte. Ceci nous ramène à la question de la catégorisation classique qui consiste à classer la traduction en deux types, soit traduction générale et traduction spécialisée. Selon Gouadec, le traducteur généraliste est polyvalent; il traduit toute sorte de document n'appartenant pas à un type particulier et ne renvoyant pas à un degré de spécialisation réel (Gouadec, 2007 : 27). Par conséquent, un traducteur généraliste devrait disposer d'une culture générale à la fois étendue et approfondie puisqu'il peut être appelé à traduire des matériaux spécialisés qu'il n'a pas le droit de refuser même s'ils requièrent un degré de compétence qu'il n'a pas 
encore atteint. Ceci pourrait être expliqué par le fait qu' «(...) il n'existe plus de véritable différence entre la traduction dite "générale » et la traduction spécialisée » (Guidère, 2010 : 117). Après avoir acquis une certaine expérience à travers la traduction de textes variés, un traducteur généraliste peut changer de trajectoire et se diriger vers la spécialité en raison de la rentabilité de ce genre de traduction et du prestige qu'elle lui confère. La distinction entre lexique général et vocabulaire spécialisé ne devrait plus avoir lieu car la frontière entre les deux est floue et a tendance à s'estomper (Guidère, 2010).

Cependant, il n'y a aucune relation entre les catégories de traduction et les méthodes utilisées pour traduire un texte donné. Le traducteur choisit ses stratégies à sa guise sans trop se soucier du domaine auquel appartient le texte source puisque un texte publicitaire peut remplir les mêmes fonctions attribuées généralement à un texte littéraire (Gile, 2005). Mais, toujours est-il que ses connaissances linguistiques ne sont souvent pas suffisantes et ne peuvent être comblées que par des connaissances extralinguistiques emmagasinées, le tout formant ce que les adeptes de la théorie du sens ont tendance à appeler bagage cognitif (Lederer, 1994 : 37). On a beau crier haut et fort que le savoir universel n'existe pas, mais rien n'est mystérieux après une recherche documentaire si on a le temps qu'il faut pour la faire. Or, le facteur temps ne joue pas toujours en faveur du traducteur des textes politiques qui est appelé à les rendre au plus vite, voire immédiatement dans certains cas.

\subsection{TRADUCTION DU DISCOURS POLITIQUE : UNE TRADUCTION SPÉCIALE}

Partant de l'hypothèse que tout texte où plusieurs terminologies sont mêlées ne pourrait appartenir à une seule discipline, soit une seule langue de spécialité, nous dirons que la traduction du discours politique est loin d'être considérée comme étant une traduction spécialisée ni générale d'ailleurs. C'est un amalgame des deux, une traduction spéciale. Spéciale parce qu'elle exige un savoir encyclopédique sans égal outre la capacité de s'adapter aux différentes idéologies qui gouvernent la forme et le contenu du produit final. En effet, l'adaptation est la stratégie la plus utilisée pour traduire ce genre de textes. Dans un discours tenu par l'ancien président égyptien Mohamed Morsi lors du sommet des pays non alignés à Téhéran en 2012, l'interprète qui traduisait ses propos en persan a remplacé le mot « syrien » par «bahreïni » à deux reprises en raison des positions politiques iraniennes pro syrienne et anti bahreïnie. De plus, une traduction spécialisée est destinée normalement à un public restreint de professionnels, contrairement aux textes politiques qui visent le grand public toutes classes sociales confondues. 
D'après la catégorisation classique, la langue politique est une langue de spécialité. Il convient de souligner que le degré de spécialisation ou de technicité dans les langues de spécialité est variable «selon les besoins de la communication, qui va de la haute technicité à la vulgarisation, en passant par la banalisation »(Messaoudi, 2010: 133). Nous croyons que le discours politique jongle entre la vulgarisation et la banalisation même si plusieurs termes sont employés par la majorité écrasante sans pour autant connaître leurs sens et les raisons en sont multiples. Selon van Dijk, le domaine de la politique est le plus inclusif comprenant l'éducation, la santé, le droit, les arts, etc. Ces derniers jouent un rôle important dans la définition des actions et du discours politiques (van Dijk, 1997 : 16). Par inclusif, il faut entendre que la politique comprend tous les domaines de la société ; elle est la pratique de gouverner un pays (Collin, 2004: 183) avec toutes ses institutions, relations extérieures, organisation et sécurité intérieures, économie, culture... etc. Ainsi, gérer un pays implique savoir ce qui se passe sur le territoire national et international, la position de son pays vis-à-vis des questions qui préoccupent l'opinion publique mais surtout savoir comment en parler. Le «comment» fait référence au vocabulaire utilisé pour discuter de ce qui est relatif aux affaires de l'Etat. Ceci dit diversité des domaines et par conséquent pluralité des langues de spécialité, le tout dans un macro domaine, si l'on peut dire, qui est la politique.

La terminologie de la langue politique est liée à la langue spéciale de la philosophie politique puisque cette terminologie assiste la formulation des questions les plus fréquentes qui concernent la relation entre l'individu et la société (Sárosi, 2014: 164). La terminologie politique remplit les fonctions suivantes :

1 - Fonction expressive, c'est-à-dire qu'elle exprime des objectifs enracinés dans la sphère réelle de la politique ;

2 -Fonction objective, c'est-à-dire qu'elle a une raison objective pour influencer la pensée et les sentiments des autres, et donc leurs actions ;

3 - Fonction symbolique, c'est-à-dire que les pensées et les sentiments sont exprimés par des symboles politiques (Sárosi, $2014: 166$ ).

Visant les masses en vue de les persuader ou dissuader, les politiciens font le tour des disciplines afin de répondre aux attentes des récepteurs dont les ambitions et les soucis sont aussi communs que divers. Ceci dit, le jargon spécialisé se transforme en un langage plus ou moins vulgarisé, banalisé par l'usage. Nous entendons souvent des termes tels que "l'enrichissement nucléaire / de l'uranium », "blanchiment d'argent», "bulle immobilière », « effet de serre », etc., et certains d'entre nous les emploient pour discuter des évènements nationaux / internationaux marquants. Cependant, parler politique 
est une chose et la comprendre en est une autre même si les médias ont contribué à la démocratisation et l'ancrage d'un langage vague perçu parfois comme une langue commune que la plupart parlent mais que peu comprennent.

\section{CONCLUSION}

En guise de conclusion, nous dirons que la traduction du discours politique serait un genre complexe; afin de bien mener sa tâche, le traducteur doit accepter le fait qu'il a besoin d'une mise à jour régulière. Cette dernière est nécessaire pour combler certaines lacunes; enrichir son vocabulaire général et spécialisé et élargir sa culture. Ces questions, bien que banales et communes à presque tout type de traduction, sont extrêmement importantes en raison de l'inclusion et la spécificité du domaine politique.

Le présent travail est loin d'être terminé, parce que nous ne pouvons pas cerner tous les aspects qui forment la richesse et la complexité d'une telle approche. En effet, cet article n'est que le début d'une future perspective de recherche dans laquelle nous nous proposons d'explorer le terrain de plus près en essayant de nous attaquer à l'analyse de la traduction de textes politiques dans d'autres langues-cultures. 


\section{RÉFÉRENCES}

Barthes, R. (1974). Théorie du texte. Encyclopedia universalis. http://www.universalis.fr/ency clopedie/theorie-du-texte/

Bielsa, E. \& Bassnett, S. (2009). Translation in Global News. London : Routledge.

Byrne, J. (2006). Technical Translation. Usability Strategies for Translating Technical Documentation. Dordrecht : Springer.

Chilton, P. \& Schäffner, C. (2002). Politics as Text and Talk: Analytic approaches to political discourse. Philadelphia : John Benjamins.

Collin, P.H. (2004). Dictionary of Politics and Government. London : Bloomsbury.

Delisle, J. (1980). L'analyse du discours comme méthode de traduction. Initiation à la traduction française de textes pragmatiques anglais. Théorie et pratique. Ottawa: Éditions de l'Université d'Ottawa.

Dorna, A. (1995). Les effets langagiers du discours politique. Hermès, 16(2), 131-146. http://hdl. handle.net/2042/15186. doi : 10.4267/2042/15186

Dubuc, R. (2002). Manuel pratique de terminologie. Québec : Linguatech.

Fairclough, N. (2001). Language and Power. New York : Longman.

Fowler, R. (1991). Language in the News: Discourse and ideology in the press. London: Routledge.

Gile, D. (2005). La traduction: la comprendre, l'apprendre. Paris: Presses Universitaires de France.

Gouadec, D. (2007). Translation as a Profession. Amsterdam/Philadelphia : John Benjamins.

Guidère, M. (2010). Introduction à la traductologie. Bruxelles : De Boeck.

Lederer, M. (1994). La traduction aujourd'hui: le modèle interprétatif. Paris : Hachette.

Lerat, P. (1988). Terminologie et sémantique descriptive. La banque des mots, numéro spécial du CTN. Paris : CILF.

Messaoudi, L. (2010). Langue spécialisée et technolecte : quelles relations ? Meta, 55(1), 127-135. http://id.erudit.org/iderudit/039607ar

Olohan, M. (2009). Scientific and Technical Translation. In M. Baker \& G. Saldanha (Eds.), Routledge Encyclopedia of Translation Studies (pp. 246-249). London \& New York: Routledge.

Riffaterre, M. (1984). Intertextual Representation: On Mimesis as Interpretive Discourse. Critical Inquiry, 11(1), 141-162.

Sárosi, K. (2014). Problems Related to the Translation of Political Texts. Acta Universitatis Sapientiae, Philologica, 6(2), 159-180.

Schäffner, C. (1997). Strategies of Translating Political Texts. In A. Trosborg, (Ed.), Text Typology and Translation. Amsterdam: John Benjamins Publishing Company.

Van Dijk, T. (1997). What is Discourse Analysis? Political linguistics, 11, 11-52. 